Kamala and Waras have been examined by Mr D Hooper,* who shows that they are very similar, but not identical.

It is worth noting that of the colouring matters examined those giving definite absorption bands yield fugitive dyes, while the others yield permanent dyes.

My thanks are due to Dr J. Gibson for assistance received during the progress of the inquiry.

\title{
Desoription of Plate.
}

(a) Colouring matter from Trichosanthes palmata, $0.5 \mathrm{~cm}$. thick.

(b)

(c) " , $2 \cdot 0 \mathrm{~cm}$. ,

(d) Chlorophyll from cabbage, $2 \mathrm{~cm}$. thick. $4.0 \mathrm{~cm}$. ,

(e) Colouringmatter fom Trichosanthes palmata + ammoniasulphideafter2days.

(f)

(g)

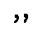

,

,

11,

(h) Chlorophyll + ammonia sulphide.

,

39,

(i) Colouring matter from Trichosanthes palmata + bydrochloric acid.

(j) Chlorophyll + hydrochloric acid.

(k) Colouring matter from Ventilago madraspatana, thin layer.

(l)

(m)

funiculus of Wattle.

thick layer.

(n) Waras.

(o) Kamala.

On a Mechanism for the Constitution of Fther. By Sir William Thomson:

(Read March 17, 1890.)

1. In a communication to the Royal Society of Edinburgh of 4th March 1889, I stated the problem of constructing a jointed model under gyrostatic domination, to fulfil the condition of having no rigidity against irrotational deformations, and of resisting rotation, or rotational deformation, with quasi-elastic forcive in simple proportion to rotation. I gave a solution, illustrated by a model, for the case of points all in one plane; but I did not then see any very simple three-dimensional solution. After many unavailing efforts I have recently found the following.

\footnotetext{
* Pharmaceutical Journal, vol. xviii. p. 213.
} 
2. Take six fine straight rods, and six straight tubes, all of the same length, the internal diameter of the tubes exactly equal to the external diameter of the rods. Joint all the twelve together with ends to one point $P$. Mechanically this might be done (but it would not be worth the doing) by a ball-and-twelve-socket mechanism. The condition to be fulfilled is simply that the axes of the six rods and of the six tubes all pass through the one point P. Make a vast number of such clusters of six tubes and six rods, and, to begin with, place their jointed ends so as to constitute an equilateral homogeneous assemblage ${ }^{*}$ of points $\mathrm{P}, \mathrm{P}^{\prime} \ldots$, each connected to its twelve nearest neighbours by a rod of one sliding into a tube of another. This assemblage of points we shall call our primary assemblage. The mechanical connections between them do not impose any constraint; each point of the assemblage may be moved arbitrarily in any direction, while all the others are at rest. The mechanical connections are required merely for the sake of providing us with rigid lines joining the points, or more properly rigid cylindric surfaces having their axes in the joining lines. Make now a rigid frame, $G$, of three rods fixed together at right angles to one another through one point $O$. Place it with its three bars in contact with the three pairs of rigid sides of any tetrahedron, $\left(\mathrm{PP}^{\prime}, \mathrm{P}^{\prime \prime} \mathrm{P}^{\prime \prime \prime}\right),\left(\mathrm{PP}^{\prime \prime}, \mathrm{P}^{\prime \prime \prime} \mathrm{P}^{\prime}\right),\left(\mathrm{PP}^{\prime \prime \prime}, \mathrm{P}^{\prime \prime} \mathrm{P}^{\prime}\right)$ of our primary assemblage. Place similarly other similar rigid frames, $G^{\prime}, G^{\prime \prime}, \& c$., on the edges of all the tetrahedrons congener $\dagger$ to the one first chosen. The points $\mathrm{O}, \mathrm{O}^{\prime}, \mathrm{O}^{\prime \prime}, \& \mathrm{c}$, form a second homogeneous assemblage related to the assemblage of $\mathrm{Ps}$, just as the reds are related to the blues in $\S 69$ of the Article referred to in the footnotes.

3. The position of the frame G-that is to say, its orientation and the position of its centre $O$ (six disposables) -is completely determined by the four points $\mathrm{P}, \mathrm{P}^{\prime}, \mathrm{P}^{\prime \prime}, \mathrm{P}^{\prime \prime \prime}$ (Thomson and Tait's Natural Philosophy, $\$ 198$; or Elements, $\$ 168$ ). If its bars were allowed to break away from contact with the three pairs of edges of the tetrahedron, we might chose, as its six co-ordinates, the six distances of its three bars from the three pairs of edges; but we suppose it to be constrained to preserve these contacts. And now let any

* See "Molecular Constitution of Matter," $\$ 45(a)$. . (j), Proc. Roy. Soc. Edin. for July 1889, to be republished as Art. XCVII. of Vol. III. of my Papers.

*Ibid., $\$ 13$. 
one of the points $\mathrm{P}, \mathrm{P}^{\prime}, \mathrm{P}^{\prime \prime}, \mathrm{P}^{\prime \prime \prime}$, or all of them, be moved in any manner. The position of the frame $G$ is always fully determinate. This is illustrated by a model accompanying the present communication, showing a single tetrahedron of the primary assemblage and a singleG frame. The edges of the tetrahedron are of copper wire sliding into glass tubes. The wires and tubes are provided with an eye or staple respectively, through which a ring passes to hold three ends together at the corners. Two of the rings have two glass tubes and one copper wire linked on each, while the other two rings have each two copper wires and one glass tube.

4. Returning now to our multitudinous assemblage, let it be displaced by stretchings of all edges parallel to $\mathrm{PP}^{\prime}$, with no rotation of $\mathrm{PP}^{\prime}$, or $\mathrm{P}^{\prime \prime} \mathrm{P}^{\prime \prime}$. This constitutes a homogeneous irrotational deformation of the primary assemblage. The frames $G, G^{\prime}, \& c$., experience merely translatory motions without any rotation, as we see readily by confining our attention to $G$ and the tetrahedron ${ }^{2} P^{\prime} \mathrm{P}^{\prime \prime} \mathrm{P}^{\prime \prime}$. Consider similarly five other displacements by stretchings parallel to the five other edges of the tetrahedron. Any infinitely small homogeneous deformation of the primary assemblage ( $\$ 1$ above) may be determinately resolved into six such simple stretchings, and any infinitely small rotational deformations may be produced by the superposition of a rotation without deformation, upon the irrotational deformation. Hence an infinitely small homogeneous deformation of the primary assemblage without rotation produces only translatory motion, no rotation, of the G frames; and any infinitely small homogeneous displacement whatever of the primary assemblage, produces a rotation of each frame equal to, and round the same axis as, the rotational component.

5. It now only remains to give irrotational stability to the $G$ frames. This may be done by mounting gyrostats properly upon them according to the principle stated in $\$ 3-5$ of Article C. of Vol. III. of my Papers, and in my Address to the Institute of Electrical Engineers, 10th January 1889. Three gyrostats would suffice, but twelve may be taken for symmetry, and for avoidance of any resultant moment of momentum of all the rotators of one frame. Instead of ordinary gyrostats with rigid fly-wheels, we may take liquid gyrostats as described below $\S 6$, and so make one very small step towards abolishing the crude mechanism of fly-wheels and 
axles and oiled pivots. But I choose the liquid gyrostat at present merely because it is more easily described.

6. Imagine a hollow anchor ring, or tore, that is to say, an endless circular tube of cireular cross-section. Perforate it in the line of a diameter, and fix into it small straight tubes to guard the perforations as shown in the accompanying diagram. Fill it with frictionless liquid, and give the liquid irrotational circuital motion as indicated by the arrow-heads in the diagram. This arrangement constitutes

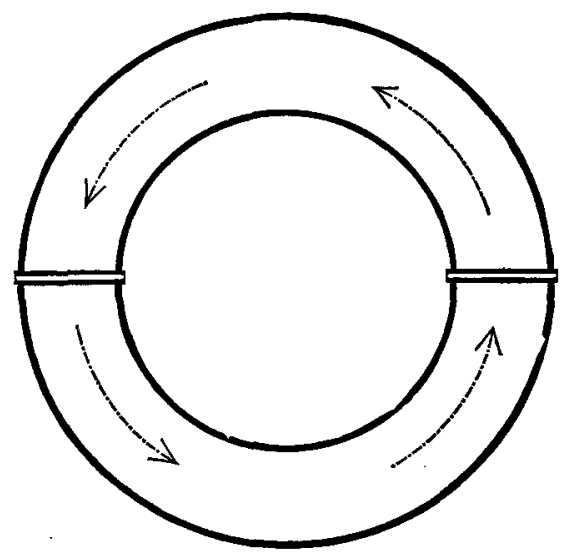

our hydrokinetic substitute for a mechanical fly-wheel. Mount it on a stiff diametral rod passing through the perforations, and it becomes the mounted gyrostat, or Foucault gyroscope, required for our models. Its use would have considerably simplified and shortened the description of a model communicated to the French Academy last September, ${ }^{*}$ which, however, was given purposely in terms of the Foucault gyroseope because it thus describes real mechanism by which the exigencies of the model can be practically realised in a very interesting and instructive manner, as may be seen in $\$ 33-5$ of Article C. of my Papers, and in my Address to the Institute. of Electrical Engineers of 10th January 1889.

7. Let $\mathrm{XOX}^{\prime}, \mathrm{YOY}^{\prime}, \mathrm{ZOZ}^{\prime}$ be the three bars of the G frame: mount upon each of them four of our liquid gyrostats, those on

* Comptes Rendus, 16th September 1889 ; Art. C. of Papers, Vol. III. 
$\mathrm{XOX}$ ' being placed as follows, and the others correspondingly. Of the four rings mounted on $\mathrm{XX}^{\prime}$, two are to be placed in the plane of $\mathrm{YY}^{\prime}, \mathrm{XX}^{\prime}$, the other two in the plane of $\mathrm{ZZ}^{\prime}, \mathrm{XX}^{\prime}$. The circuital fluid motions are to be in opposite directions in each pair.

8. The gyrostatic principle stated in $\$ 5$ of Art. C. of Vol. III. of my Papers, applied to our G frame, with the twelve liquid gyrostats thus mounted on it, shows that if, from the position in which it was given with all the rings at rest, it be turned through an infinitesimal angle $i$ round any axis, it requires, in order to hold it at rest in this altered position, a couple in simple proportion to $i$; and that this couple remains sensibly constant, as long as the planes of all the gyrostats have only changed by very small angles from parallelism to their original directions. Hence, with this limitation as to time, our primary homogeneous assemblage of points, controlled by the gyrostatically dominated frame, $G, G^{\prime}$, \&c., fulfils exactly the condition stated for the ideal ether of $\$ 14$ of Art. XCIX. of Vol. III. of my Papers, which is as follows:- It has no intrinsic rigidity, that is to say, no elastic resistance to change of shape; but it has a quasi-rigidity, depending on an inherent quasi-elastic resistance to absolute rotation. It is absolutely non-resistant against change of volume and against any irrotational change of shape. Or it is absolutely incompressible. The model may be made so by introducing struts between the points $\mathrm{P}$ of the primary assemblage and their nearest neighbours $\mathrm{O}, \mathrm{O}^{\prime}$, \&c., of the $\mathrm{G}$ frames according to $\S 70$ of "Molecular Constitution of Matter" (Proc. Roy. Soc. Edin., July 1889).

9. If the velocity of the motion of the liquid in each gyrostat be infinitely great, each $G$ frame exerts infinite resistance against rotation round any axis; and if the bars and tubes constituting the edges of the tetrahedron, and the bars of the $G$ frames were all perfectly rigid, the primary assemblage is incapable of rotation or of rotational deformation; but if there is some degree of elastic flexural yielding in the edges of the tetrahedron, or in the bars of the $G$ frame, or in all of them, the primary assemblage fulfils the definition of $\S 9$, without any limit as to time, that is to say, with perfect durability of its quasi-elastic rigidity.

10. A homogeneous assemblage of points with gyrostatic quasirigidity conferred upon it in the manner described in $\$ \S 2-8$ would, 
if constructed on a sufficiently small scale, transmit vibrations of light exactly as does the ether of nature. And it would be incapable of transmitting condensational-rarefactional waves, because it is absolutely devoid of resistance to condensation and rarefaction. It is, in fact, a mechanical realisation of the medium to which $I$ was led one and a half years ago, ${ }^{*}$ from Green's original theory, by purely optical reasons, in endeavouring to explain results of observation regarding the refraction and reflection of light.

\section{On the Swimming Bladder and Flying Powers of Dactyl- opterus volitans. By W. L. Calderwood. Communicated by Professor Ewart. (With Plate.)}

(Read February 17, 1890.)

Dactylopterus volitans, the so-called "flying gurnard," is not included by Günther in the genus Triglidæ, as its name might imply, but is assigned to the small allied family of Cataphracti.

Guinther diagnoses it as follows :-- "Dactylopterus, no lateral line, pectoral fins very large, an organ of flight, with the upper portion detached and shorter, granular teeth in the jaws, none on the palate, air bladder divided into two lateral halves, each with a large muscle."

Before proceeding to describe in detail the swimming bladder and other anatomical peculiarities, it may be well to state, that the skull is provided with a superficial bony covering which projects backwards over the region of the "shoulder" in two flattened plates, each terminating in a spine (seen in fig. 2). Also that the first four vertebræ of the column have coalesced so as to form a rigid tube, the neural spines being united as a vertical plate, which for convenience I have termed the neural plate.

The swimming bladder, so far as I am aware, has a unique position, since it is situated not below, but above the vertebral column, not forming part of the abdominal contents, but situated dorsally in a special cavity of its own. The appearance of the

* Philosophical Magazine, Nor. 1888, "On the Reflection and Refraction of Light," by Sir W. Thomson. 